\title{
Cementación de revestidor en flujo de gas/agua presurizado superficial no esperado en un campo de desarrollo costa afuera: Rio de Janeiro, Brasil
}

\section{Casing cementing in shallow pressurized gas/water flow not expected in a field development offshore: Rio de Janeiro, Brazil}

\author{
Francisco Mavares ${ }^{1}$, Alberto Pertuz ${ }^{2}$ \\ ${ }^{1}$ Escuela de Ingeniería Mecánica, Facultad de Ingeniería, Universidad Central de Venezuela. Código Postal N 48222, Los \\ Chaguaramos, Caracas, Venezuela. Email: fmavares@hotmail.com \\ ${ }^{2}$ Escuela de Ingeniería Mecánica, Universidad Industrial de Santander, Bucaramanga, Santander, Colombia. \\ Email: apertuzc@uis.edu.co
}

RECIBIDO: Febrero 12, 2017. ACEPTADO: Marzo 30, 2017. Versión FinAL: Mayo 24, 2017.

\begin{abstract}
RESUMEN
El siguiente estudio está enfocado en la estrategia sugerida para atacar un problema de flujo de gas/agua presurizado superficial no esperado en revestidores que son perforados y cementados sin el equipo de control de pozo en campos costa afuera. Se analizan casos históricos donde las presiones anormales de las arenas de agua observadas en el registro de resistividad no fueron identificadas en la herramienta PWD (Por sus siglas en Ingles, "Pressure While Drilling"). El evento de flujo fue observado por el vehículo operado remotamente. Se sugiere un árbol de decisiones a la hora de un flujo de gas/agua presurizado superficial no esperado. Eventos de este tipo ocasionan una situación de flujo descontrolado colocando en riesgo la seguridad de las personas que trabajan en la unidad marítima de perforación, la misma unidad y pérdidas económicas por la ejecución de operaciones no planificadas.
\end{abstract}

PALABRAS CLAVE: Flujo de gas/agua presurizado superficial, Fuerza gel, Revestidor superficial, Resistencia compresiva, Cementación de revestidores.

\begin{abstract}
The following study is focused on the suggested strategy to attack a problem of Shallow Gas/Water flow in drilled and cemented casing without equipment to prevent blowouts in offshore fields. We analyze historical cases where abnormal pressure water sands observed in the resistivity log were not identified in the Pressure While Drilling tool. The event flow was observed by the remotely operated vehicle. We suggest a decision tree when a shallow gas/water flow is unexpected. Such events cause an uncontrolled flow situation by putting at risk the safety of persons working in the maritime unit drilling, the same unit and economic losses for the execution of unplanned operations.
\end{abstract}

KEYWORDS: Shallow Gas/Waters Flows, Gel Strength, Surface casing, Strength compressive, Casing cement.

\section{INTRODUCCIÓN}

En campos de desarrollo es poco común observar arenas presurizadas superficiales en revestidores donde normalmente esta fase es perforada alrededor de $1000 \mathrm{~m}$ por debajo del lecho marino. Los revestidores conductor $\mathrm{y}$ de superficie son perforados con retorno al lecho marino y el equipo de control de pozo todavía no está instalado por lo que un eventual flujo de gas o agua se torna incontrolado, al tratarse de una situación no esperada, normalmente no se cuenta con los recursos para llevar a cabo una operación de cementación que se base en un sistema cementante de flujo de gas/agua presurizado superficial, por tanto diseñar un plan de contingencia es imperativo para ejecutar esta operación de manera segura. 


\section{ANTECEDENTES}

Si ocurre el flujo, hay que evaluar los riesgos y costos de continuar con la perforación bajo balance versus controlarlo con largos volúmenes de fluido pesado. En algunos casos históricos han utilizado sistemas cementantes espumados en revestidores que atraviesan zonas presurizadas superficiales. Entre los beneficios tenemos: mejora de la eficiencia de desplazamiento, control de pozo por el hecho de estar un fluido expansivo durante la fase de transición de un sistema cementante líquido a uno con suficiente resistencia al gel que contenga el flujo y fácil colocación en la unidad marítima de perforación. [8]

Luego de colocar el sistema cementante, la caída de la presión hidrostática durante el desarrollo del esfuerzo de gel, en condición estática resulta en bajo balance, pudiendo inicial el flujo de gas/agua presurizada superficial. Determinando el tiempo de desarrollo del esfuerzo de gel estático del sistema cementante, calculando la perdida de presión en el anular y el instante donde la presión de poros de la arena de gas / agua superficial excede la presión hidrostática del anular, el esfuerzo de gel del sistema cementante puede ser anticipado [4][9][10].

En la región del delta del Nilo no fue la excepción de los riesgos de perforar en áreas con flujos de gas y agua observados en la mayoría de los proyectos de perforación costa afuera en el mundo. Una consecuencia de un flujo presurizado superficial no esperado es una situación de descontrol del pozo, donde en los revestidores superficiales todavía no se ha instalado el equipo de control de pozo. En algunos casos reportados, el flujo ha sido controlado antes de correr el revestidor, en otros ocurre después de la cementación del revestidor de superficie. Cada una de estas operaciones le costó al operador entre 1.5 y 4 millones de dólares para solucionar el problema. Una preparación adecuada del pozo, un correcto diseño del lodo de perforación y un sistema cementante espumado han sido soluciones efectivas. Adicionalmente, avances realizados en los sistemas cementantes para situaciones costa afuera donde hay un reducido margen entre los gradientes de poros y fractura, bajas temperaturas del lecho marino e impredecibles flujos superficiales son factores adversos al diseño de un sistema cementante. Las consecuencias de un flujo de gas/agua presurizado superficial pueden ser tan severas como la pérdida del pozo. Una de las dificultades en evaluar un flujo de gas/agua presurizado superficial, es que esta puede no ocurrir durante las operaciones de perforación o cementación, pero puede ocurrir horas después del trabajo de cementación, cuando el sistema cementante no ejerce una presión hidrostática competente en la formación. Este puede ser el camino donde el gas o el agua atraviesen el cemento hasta la superficie. En zonas donde la profundidad de agua es mayor a $600 \mathrm{~m}$, el gradiente de fractura a la profundidad del revestidor conductor, no soporta el peso de un sistema cementante convencional y pérdidas de circulación son observadas. Las soluciones en estos casos son el uso de un sistema cementante a base de micro-esferas, aditivos de bajo peso o uno espumado en el rango de 10 a $12 \mathrm{lpg}$. Cementar un revestidor de superficie en una región de riesgo de flujo de gas /agua presurizado superficial en el Delta del Nilo ha sufrido grandes cambios. Primero se verifica el riesgo de flujo presurizado superficial, en las proyecciones sísmicas, en casos donde hay una probabilidad baja y el pozo necesita ser perforado en esa locación, se perfora un hoyo piloto de 12-1/4". Se requerirá de una locación alternativa si el flujo es detectado. Una prueba corta de flujo de 30 minutos, donde el pozo es observado en condiciones estáticas para eliminar los efectos de la densidad equivalente de circulación que enmascara el potencial del pozo a fluir bajo condiciones dinámicas. Estos pozos son controlados con lodo de perforación entre 11 y 13 lpg, para ayudar a mantener la estabilidad del pozo cuando es bajado el revestidor de superficie. El mecanismo de zonas presurizadas superficiales se puede predecir y se debe tomar precauciones durante el diseño del sistema cementante y en la perforación [5].

Una solución para verificar flujos de gas / agua presurizadas superficiales, que tienen baja probabilidad de ocurrir, según los registros sísmicos, es la de perforar un hoyo piloto de 12-1/4" hasta la profundidad de asentamiento de la zapata del revestidor de superficie, casos históricos son mencionados a continuación: fue perforado un hoyo piloto de 12-1/4" y detectada una zona a $692 \mathrm{~m}$ con $5 \%$ de probabilidad de agua presurizada superficial. Fue analizada la posibilidad de colocar la zapata de 20 " antes de $692 \mathrm{~m}$ y colocar un liner de 16 " en frente de esta zona, con equipo de control de pozo; que al compararlo con un sistema cementante adecuado, colocando la zapata de 20 " a $1000 \mathrm{~m}$, se decidió por la segunda opción. El diseño del sistema cementante fue basado en: sistema estabilizado, baja pérdida de fluido, baja permeabilidad (con la utilización de microsílica alrededor de 0,15 micrón), tiempo de tránsito para desarrollar el esfuerzo de gel estático (cemento clase c, con alto contenido de calcio) y minimizando la reducción de volumen. Las causas de un flujo después de un trabajo de cementación pueden ser que la presión hidrostática en el anular disminuye con respecto a la presión de formación por deshidratación, agua libre o canales o durante la gelificación del sistema cementante [11].

Las operaciones costa afuera en el Delta del Nilo se ejecutan en profundidades de agua entre 200 y $2600 \mathrm{~m}$, las consecuencias son inesperadas con fluidos 
incontrolados (reventones) sin equipo de control de pozo instalado. En algunos casos el flujo fue controlado antes de la bajada del revestidor, siendo observado después de la cementación. Cementos espumados de baja densidad y alta resistencia, han sido usados en un margen estrecho entre los gradientes de poro y fractura, además con secciones perforadas con potenciales flujos. Sistemas cementantes espumados han sido efectivos en muchos casos. Diseños en ambientes de gradientes de fracturas bajos, en profundidades de agua de $300 \mathrm{~m}$, donde flujos no fueron observados, es posible cementar el revestidor de superficie utilizando un sistema cementante convencional, que consta de un sistema cementante extendido de llenado de 12 a $12.5 \mathrm{lpg}$ a base de bentonita prehidratada, en la parte alta del revestidor y uno de cola de peso $15.8 \mathrm{lpg}$, que se coloca en la parte baja, alrededor de la zapata. En profundidades de agua mayores a $600 \mathrm{~m}$, el gradiente de fractura baja y el peso de los sistemas cementantes convencionales, no es soportado por la formación resultando en perdida. Opciones para este caso es un sistema cementante de bajo peso a base de microesferas o cementos espumados en el rango de 10 a $12 \mathrm{lpg}$ [1].

Para el pozo exploratorio titán nro. 1, el equipo de perforación seleccionó un sistema cementante espumado con nitrógeno de corto tiempo de tránsito. El tiempo de transito es generalmente entendido como el intervalo de tiempo en el que el sistema cementante desarrolla suficiente esfuerzo de gel para impedir el flujo de fluidos a través del cemento. Utilizando un sistema cementante de corto tiempo de tránsito, minimiza el tiempo en que el sistema cementante es vulnerable a canalización. Usando nitrógeno la densidad del sistema cementante es compatible con la ventana entre los gradientes de poro $\mathrm{y}$ fractura, al mismo tiempo desarrollan excelentes resistencias compresivas [13].

Los bloques ubicados en la Plataforma Deltana (Venezuela), están influenciados por bolsones de gas superficiales, que se comunican con yacimientos presurizados profundos, a través de fallas que pueden originar reventones durante la perforación o cementación de los pozos. Durante la perforación del pozo loran-1x, en la campaña de perforación exploratoria de los años 80 , se obtuvo una migración de gas a través del espacio anular, una vez cementado el revestidor superficial, esto llevo a la movilización inmediata de la unidad marítima de perforación, desde la localización en dirección opuesta a las corrientes marinas con la finalidad de evaluar diariamente el comportamiento del pozo. Este evento tuvo un tiempo perdido de 15 días, incluyendo el alto riesgo de exposición del personal a sufrir accidentes fatales, perdida del pozo y/o perdida de la unidad marítima de perforación. Las dos primeras fases de los pozos submarinos se ejecutan con retorno al lecho marino, sin utilizar el equipo de control de pozo. Las mayores pérdidas humanas y materiales en costa afuera se han generado como consecuencia de la ocurrencia de arremetidas de gas en zonas superficiales. Por ejemplo, entre los años 1987 y 1988, los reventones causaron pérdidas en plataformas costa afuera por el orden de los 200 MM USD.

Con la finalidad de minimizar los riesgos operacionales al perforar zonas con gas presurizada superficial, se decidió corroborar la interpretación sísmica en las correspondientes localizaciones de los pozos, mediante la aplicación de técnicas tales como: la perforación de un hoyo piloto y ampliado sin conductor marino, utilizando lodo densificado mediante la dosificación de un mezclador dinámico de fluidos, la toma de información mientras se perfora en tiempo real de emisiones de rayos gamma, conductividad y diferencial de presión anular en el hoyo piloto. Se visualizó retorno de fluido y gas al lecho marino durante la perforación del hoyo piloto de 8$1 / 2$ " en el pozo bor-1se, al finalizar la perforación la tendencia del gas disminuyó, aun así, se decidió dejar fluido densificado de 9,3 lpg en el hoyo y mantener las propiedades anti-migratorias de la lechada. En función de las simulaciones superficiales y considerando el riesgo a producirse perdida de circulación durante la cementación, se decidió bajar la densidad de la lechada a de peso inicial de 13,5 y 15,6 a 12,5 lpg, esto considerando el uso de una lechada liviana extendida con micro-esferas huecas y aditivos sólidos inertes para mantener una resistencia a la compresión aceptable en temperaturas de circulación que van desde 60 a $80^{\circ} \mathrm{F}$, una resistencia a la compresión de 500 lbs a las 24 horas, que resista los esfuerzos de las fases siguientes y del peso del equipo de control de pozo, el cual pesa alrededor de $570.000 \mathrm{lbs}$. El filtrado menor que $35 \mathrm{cc} / 30$ minutos permitió minimizar la pérdida de fluido de la lechada, reduciendo el riesgo de disminución del tiempo de espesamiento y la posible migración de gas a través de la matriz del cemento. En un primer diseño se trató de reducir el filtrado a $25 \mathrm{cc} / 30$ minutos, lo cual originó un aumento considerable en la viscosidad del sistema cementante. Como factores primordiales de migración de gas, se consideraron un tiempo de gelificación de la lechada en condición estática menor que 25 minutos, una resistencia al gel en el intervalo de 100 a $500 \mathrm{lbs} / 100$ pies2 y un tiempo menor a 30 minutos en el desarrollo de 30 UC a 70 UC en la curva de tiempo de transición. Se consideró agua libre cero para minimizar cualquier decantación de sólidos en la lechada y así evitar desbalance hidrostático durante la colocación del cemento [3].

Aplicando el modelo de pérdida de presión hidrostática introducido por Sabins, et al, en 1980 [12], y luego aplicado por Mueller [2] para cuantificar la perdida de 
presión hidrostática en escenarios de flujos de gas/agua presurizadas superficiales, el esfuerzo de gel estático crítico para un sistema cementante en frente de un acuífero de presión anormal. Sistemas cementantes de bajo peso son usados en aguas profundas en áreas con riesgo geológico típicamente reduciendo la relación agua del sistema cementante y con un corto tiempo de tránsito, normalmente sistemas espumados o con micro esferas huecas, requerimientos para flujo de gas / agua presurizada superficial en escenarios de incertidumbre del gradiente de poro y fractura, en ambiente de bajas temperaturas. Las micro-esferas huecas son mezcladas por aditivos sólidos y son formulados para una densidad específica, desviaciones en la densidad resultan en alteraciones de sus propiedades críticas. Sistemas espumados son obtenidos a través de la adición de nitrógeno, material inerte, la densidad puede variar cambiando la relación gas / liquido sin afectar las propiedades del sistema [7].

\section{MARCO TEÓRICO}

\subsection{Concepto de cementación primaria}

Es el proceso de colocar un sistema cementante con propiedades adecuadas en el espacio anular entre el revestidor y el pozo abierto, para tener aislamiento zonal en las áreas de interés.

\subsection{Objetivos de las cementaciones en aguas profundas}

$\checkmark$ Sellar el espacio anular y obtener un buen sello hidráulico. Evitando la migración de fluidos entre las diversas zonas permeables en el espacio anular.

$\checkmark$ Remover los ripios de perforación y remplazarlo por cemento.

$\checkmark$ Proveer soporte, resistencia del revestidor y soporte a los revestidores posteriores.

$\checkmark$ Control de pérdida de fluido.

$\checkmark$ Por medio de un frague rápido del cemento, prevenir posible migración de gas en el pozo.

$\checkmark$ Proteger el revestidor de los esfuerzos y choques cuando se perfora para profundizar.

$\checkmark$ Proteger el revestidor de la corrosión.

$\checkmark$ Proteger al revestidor de los esfuerzos externos que pudieran producir colapso.

$\checkmark$ Proteger al revestidor contra presiones internas que pudieran generar estallido.

$\checkmark$ Proteger al revestidor durante los trabajos de cañoneo.

$\checkmark \quad$ Aislar las zonas con pérdidas de circulación.

$\checkmark$ Proteger la(s) formación (es) contra derrumbes.

$\checkmark$ Sellar zonas no productivas. $\checkmark$ Aislar las zonas con pérdidas de circulación.

$\checkmark$ Proteger la(s) formación (es) contra derrumbes.

$\checkmark$ Sellar zonas no productivas.

$\checkmark$ Sellar las aguas de las formaciones productivas.

$\checkmark$ Sellar zonas de gas en caso necesario.

$\checkmark$ Cumplir con requerimientos de seguridad, viabilidad económica y gubernamental.

$\checkmark$ Garantizar el aislamiento zonal durante la vida productiva del pozo, considerando trabajos de reacondicionamiento, cañoneo, estimulación química y de fracturamiento hidráulico, inclusive, el abandono del pozo.

\subsection{Flujo de gas / agua presurizada superficial}

Concepto: es un flujo de gas / agua por los alrededores del revestidor hacia el lecho marino o la superficie terrestre. Este flujo de agua puede erosionar el soporte estructural del pozo hasta producir un doblez del revestidor hasta ocasionar su falla, lo que puede comprometer su integridad y como resultado tener una pérdida de control del pozo.

Se han identificado cuatros mecanismos que causan flujo de agua presurizada superficial:

\subsubsection{Fracturas inducidas.}

En este mecanismo la presión generada a la profundidad de la zapata excede el esfuerzo de la formación causando una fractura que provee un canal para que fluya el agua hasta el lecho marino, generando pequeños volcanes en la superficie en la que se pueden escapar fluidos y granos de formación. Normalmente ocurre en los revestidores superficiales de 20", 16" o 13-3/8". Esta presión adicional para llegar a la fractura puede ser de fricción, empaquetamiento del pozo, ripios en suspensión o incremento del peso del lodo. Estas secciones son perforadas sin el equipo de control de pozo, con retorno al lecho marino. Ninguna arena necesita estar presente para ocurrir esta falla.

\subsubsection{Almacenamiento inducido.}

En esta condición la presión generada por la columna de lodo carga una formación permeable y porosa que estuvo normalmente presurizada. Este fenómeno es común en las primeras camadas de sedimentos del suelo marino. Este mecanismo ocurre normalmente entre el revestidor conductor y de superficie que son perforados con retorno al lecho marino, cuando la circulación es detenida, la formación se carga debido a la alta presión en el pozo, resultando en flujo en los alrededores de la cabeza de pozo, observándose esto con el vehículo operado remotamente (ROV, por sus siglas en inglés "Remote Operated Vehicle"). En principio el flujo es muy fuerte y significativo, pero con el tiempo declina rápidamente hasta observar una nube en los alrededores del pozo. En 
casos severos el flujo puede durar una hora hasta aparecer la nube, la cual puede estar presente durante días o semanas. Normalmente se perfora el pozo donde se colocará el revestidor conductor, con agua de mar y con bombeo constante de píldoras viscosa para acarrear los ripios hasta el lecho marino, luego de perforado hasta la profundidad final se deja un fluido pesado que controle al pozo y se deja en condiciones estáticas hasta la instalación del revestidor conductor. Cuando se decide perforar el pozo y se aguarda por cemento para bajar el revestidor, puede ser una causa de presurizar estas zonas porosas y permeables.

\subsubsection{Arenas geo-presurizadas en el intervalo del conductor.}

Perforar arenas geo-presurizadas antes de correr el equipo de control de pozo y la columna de riser es la causa más común de flujo de agua superficial y uno de los mecanismos más peligrosos. Cuando arenas geopresurizadas son encontradas antes de la corrida del revestidor de superficie, del equipo de control de pozo y de la columna de riser, las prácticas convencionales de control de pozos no son posibles. Algunas operadoras consideran perforar las secciones con retorno al suelo marino con fluido pesado, pero esta opción no es práctica por las dificultades de logística y altos costos de los grandes volúmenes requeridos. Cuando una formación geo-presurizada no es contenida, ella fluye llevando sólidos de formación hasta el lecho marino con el consecuente alargamiento del pozo y formación de cráteres alrededor de la cabeza de pozo. Si se decide cementar bajo estas condiciones, el sistema cementante será contaminado y formará canales posiblemente hasta la superficie del suelo marino, poniendo en peligro la fundación del pozo. Cuando se perfora formaciones geopresurizadas, algunos ripios incrementan el peso del agua de mar, que pueden suprimir el flujo, si este se incrementa, fluido más pesado es desplazado. Si se induce almacenaje el flujo de una formación geopresurizada se incrementa con el tiempo.

\subsubsection{Transmisión de geo-presión a través de canales de cemento.}

Una pobre cementación puede ser causa de flujo de agua presurizada superficial por el revestidor de superficie o conductor. La geo-presión puede ser transmitida por canales luego de ejecutada la cementación en el pozo. Este mecanismo puede aparecer tarde en la vida del pozo. La presión hidrostática de la columna del fluido de perforación contiene la formación hasta que los sólidos decantan, resultando en un fluido más liviano con la subsecuente transmisión de la sobre-presión.

\subsection{Esfuerzo de gel estático}

Se requiere un rápido desarrollo de la resistencia de gel estático en un intervalo de tiempo para pasar de 100 a 500 lbs/100 pies2. Este concepto considera un intervalo de tiempo de 30 minutos para un flujo severo y 45 minutos para uno mediano, tiempo que según experimentos es suficiente para evitar que el gas atraviese el espacio anular cementado.

MPR: $\quad$ SGS

$300 \times$ (L/Def)

Donde:

MPR: restricción de presión máxima, en lbs.

SGS: $\quad$ esfuerzo de gel estático, en lbs/100

pies $^{2}$.

1: $\quad$ longitud de la columna de cemento, en pies.

Def: diámetro efectivo de la columna de cemento, en pulgadas (diámetro de hoyo - diámetro de revestimiento).

La ecuación 1 es usada para predecir la presión diferencial que debe ser aplicada para romper gel y establecer la circulación en lodos gelificados ubicados en el espacio anular. Despejando el esfuerzo de gel estático:

SGS: MPR x 300 x (1/Def).

El esfuerzo de gel estático es medido en un consistómetro a baja rotación, con la rampa de temperatura y presión del pozo, que convierten la ultra baja velocidad rotacional, aproximadamente $0,1 \mathrm{rpm}$ para determinar el esfuerzo de gel. El resultado es graficar el tiempo contra la viscosidad, en unidades de consistencia bearden en lbf/100 pies $^{2}$. El método ultrasónico predice el esfuerzo de gel estático contra el tiempo, usando un algoritmo. El sistema cementante debe tener un esfuerzo de gel inicial alto.

\subsection{Presión de poros}

Es la presión de fluidos contenido en los espacios porosos de la roca. En una roca solo parte del volumen total es ocupado por partículas sólidas, que se acomodan formando una estructura. El volumen restante es llamado de poros, ocupado por fluidos. Para formaciones sedimentarias es común utilizar valores de 8,5 a 9 lpg, para valores de masa específicas de agua de formaciones. Para gas un valor comúnmente utilizado es 2 lpg y para petróleo se utiliza 7 lpg [5]. 


\subsection{Presión de fractura}

Es la presión que lleva a la falla de la roca por tracción, de la misma forma que ocurre para el colapso de la formación, la fractura puede ocurrir en función de un bajo peso del fluido de perforación, llevando a una falla debido a la fractura inferior o por alto peso de fluido de perforación, ocurriendo una falla por fractura superior. Las consecuencias operacionales son la perdida de fluido de perforación para la formación, conocida como perdida de circulación [6].

\subsection{Ventana operacional}

Es la variación permitida por la presión ejercida por el fluido de perforación dentro del pozo, de forma de mantener la integridad de este, respetando las presiones de poros, fractura y colapso. El límite inferior es establecido por el mayor valor entre las presiones de poros y colapso inferior. El límite superior es establecido por la curva de presión de fracturas o colapso superior [6].

\subsection{Problemas encontrados en revestidores superficiales}

$\checkmark \quad$ Flujo de gas/agua presurizado superficial.

$\checkmark \quad$ Baja temperatura en los revestidores conductor y de superficie.

$\checkmark$ Riesgo de pérdida de circulación en zonas superficiales.

$\checkmark$ Baja velocidad durante la colocación del cemento al espacio anular.

$\checkmark$ Bajo desplazamiento del lodo por el cemento, alta posibilidad de canalización debido a la geometría del hoyo/revestidor y a los bajos caudales de bombeo del sistema cementante.

\subsection{El flujo de agua superficial ocurre en:}

$\checkmark \quad$ Zonas sobre-presurizadas de alta permeabilidad.

$\checkmark$ Arenas y arcillas no consolidadas.

$\checkmark$ Fallas.

$\checkmark \quad$ Margen estrecho entre la presión de poro y de fractura.

$\checkmark$ Entre 150 y $1100 \mathrm{~m}$, por debajo del lecho marino.

$\checkmark \quad$ En formaciones con un rango de 100 md a 10 darcys de permeabilidad.

$\checkmark \quad$ Rangos de temperaturas bajas de 45 a $90{ }^{\circ} \mathrm{F}$.

\subsection{Problemas de los flujos de agua superficiales}

$\checkmark \quad$ Flujo durante la perforación o cementación.

$\checkmark \quad$ Perdida de circulación durante la perforación o colocación del cemento.

$\checkmark$ Grandes zonas lavadas.

$\checkmark$ Arrumbamiento de las zonas vecinas al hoyo, disminuyen la integridad estructural del pozo, que debiera soportar el peso del equipo de control de pozo y de la columna del riser.

$\checkmark$ Perdida del pozo y/o de la unidad marítima de perforación.

\subsection{Efecto de la baja temperatura en los sistemas cementantes}

$\checkmark \quad$ Tiempo de frague muy largos.

$\checkmark \quad$ Lento desarrollo de la resistencia compresiva.

$\checkmark$ Estimación de la temperatura API no es aplicable en pozos costa afuera, normalmente se utilizan simuladores térmicos para estimarla.

\subsection{Fases de un sistema cementante hasta la fase de fragüe}

En la figura 1, observamos las etapas de un sistema cementante luego que es colocado en el espacio anular, primero se encuentra en estado líquido, luego pasa por un proceso de gelificación donde baja levemente la presión hidrostática, luego pasa por el proceso de hidratación donde baja aún más la presión hidrostática con una pendiente de descenso más fuerte, que inclusive llega a niveles inferiores que la presión de poros, punto crítico, ya que es justo en este momento cuando el fluido de formación puede invadir el espacio anular, contaminando el sistema cementante, atravesando el mismo y generando canales. Por último, tenemos el periodo de fragüe donde el sistema cementante pasa al estado sólido.

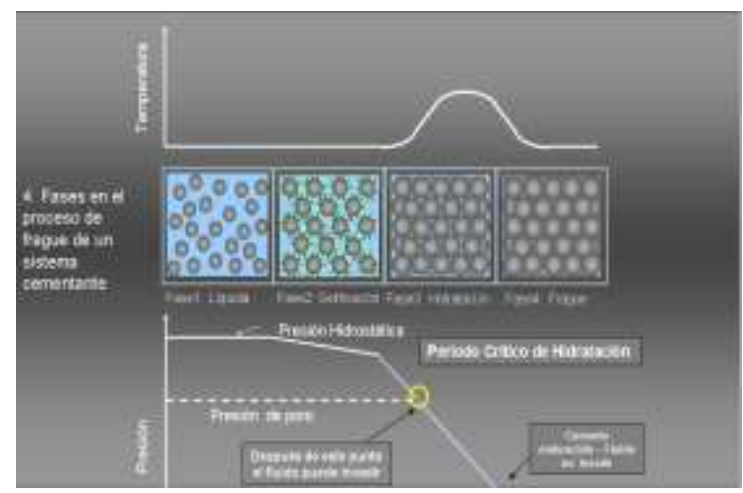

Figura 1. Fases de un sistema cementante. Fuente: Schlumberger. 
En caso de existir un flujo de gas o agua presurizada superficial, el efecto de las bajas temperaturas en la fase perforada hace que el periodo crítico de hidratación se extienda, aumentando de este modo el periodo donde la presión hidrostática está por debajo de la presión de poros, como se observa en la figura 2.

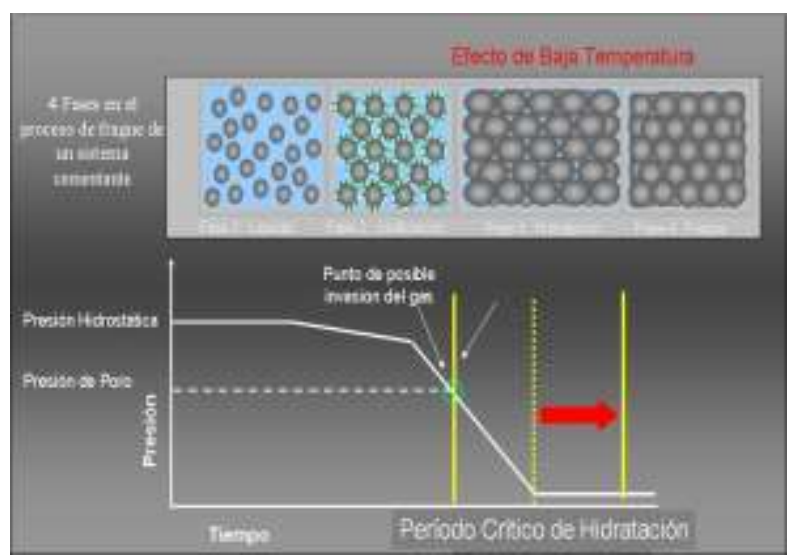

Figura 2. Efecto de baja temperatura en las fases de un sistema cementante. Fuente: Schlumberger.

En flujos de gas o agua presurizado superficial se recomienda que el intervalo en desarrollar un esfuerzo de gel estático de 100 a $500 \mathrm{lbs} / 100$ pies 2 este en un periodo de tiempo de 30 minutos para el caso de gas o agua severo, y de 45 minutos para el caso moderado, ver la figura 3 .

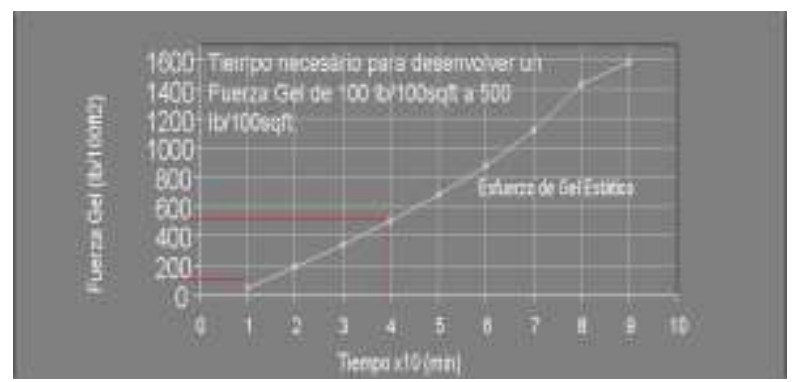

Figura 3. Esfuerzo de Gel Estático. Fuente: Prueba de Laboratorio.

\section{CASOS HISTÓRICOS}

\subsection{Caso 1. 13-3/8" Slender}

En un campo de desarrollo a una profundidad de agua de $685 \mathrm{~m}$, un revestidor conductor de 30 " fue colocado a 716 $\mathrm{m}$. Seguidamente fue perforado un hoyo de 17-1/2" a 1613 m, ver Figura 4. Ningún indicio de flujo de agua presurizada superficial fue evidenciado durante la perforación ni en el registro de medición de presión en el anular mientras se perfora (PWD, por sus siglas en inglés,
"Pressure While Drilling"). El registro de resistividad evidenció unos lentes de arenas contenedoras de agua. Fue colocado un fluido salino tratado con almidón (STA) de peso 9,7 lpg y aguardado por llegada de cemento a bordo. El pozo fue reacondicionado y colocado un nuevo fluido STA de 9,7 lpg. Fue bajado el revestidor de 13$3 / 8$ " con una columna interna (stinger) y asentado con una columna de asentamiento. No fue evidenciado flujo durante la corrida del revestidor y asentamiento del alojador de alta de 16-3/4" en el alojador de baja 18-3/4". Fue circulado para cementar con agua de mar durante 3 horas, al parar la circulación para probar la línea que conecta el pozo con la unidad de cementación, fue observado un flujo en los alrededores de la cabeza de pozo. Se verificó las bombas de la unidad marítima de perforación y de cementación fuera de operación. Se evidenció flujo en los alrededores de la cabeza de pozo con el vehículo operado remotamente, la suspensión llegó hasta $300 \mathrm{~m}$. Se colocó el peso del revestidor, stinger y de la columna de asentamiento en el bloque viajero para evitar que la cabeza de pozo se hundiera.

En la figura 4, se observa el diseño de revestidores del pozo, en la figura 5 , se observan donde se encontraron los lentes de arena desde 900 hasta $1534 \mathrm{~m}$.

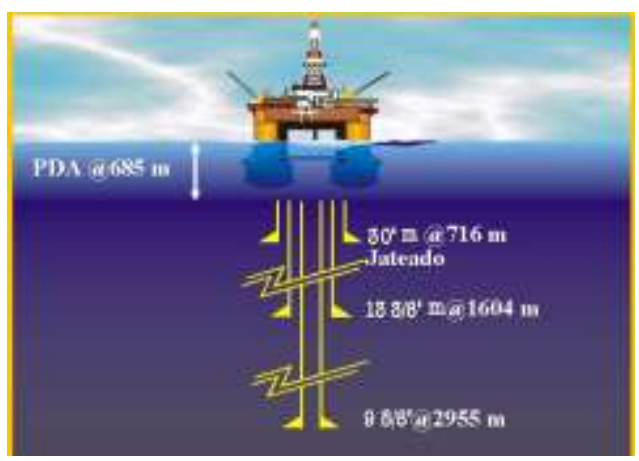

Figura 4. Esquema de pozo Caso 13-3/8" Slender. Fuente: Elaboración propia.

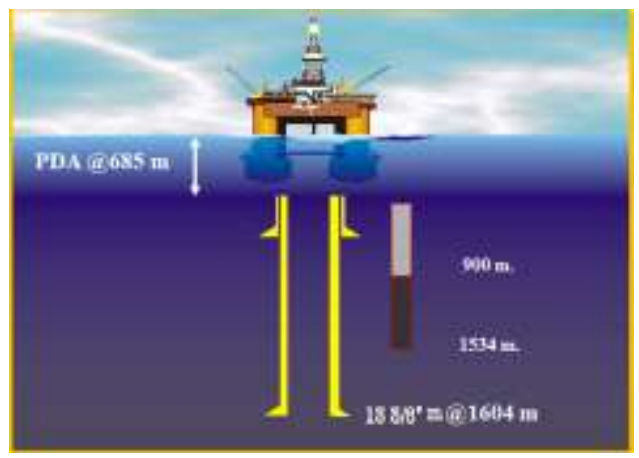

Figura 5. Arenas de agua encontrados durante la perforación caso 13-3/8" Slender. Fuente: Elaboración propia. 


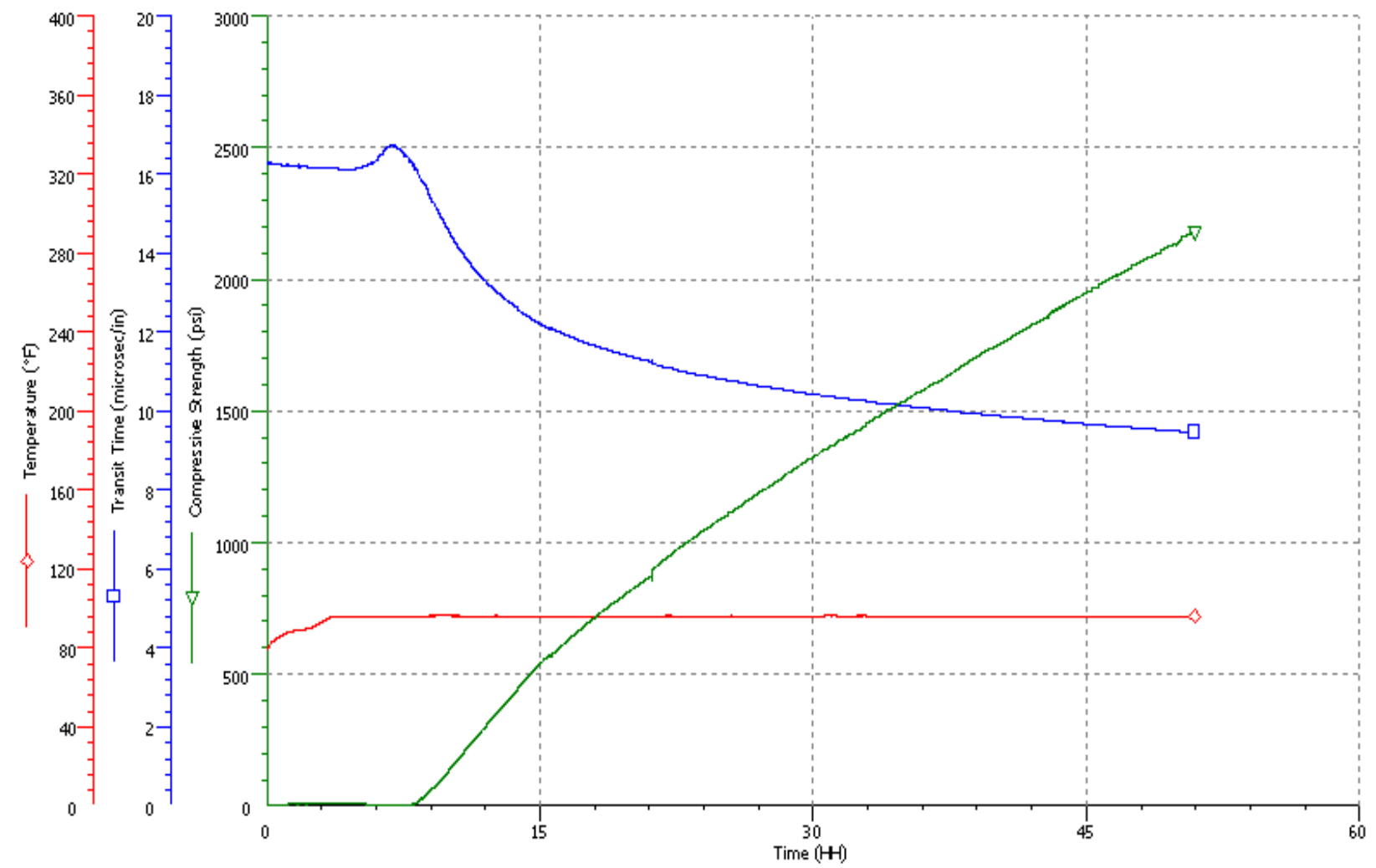

Figura 6. Prueba de Lavoratorio de Resistencia compresiva. Fuente: Prueba de Laboratorio.

En la figura 6 se observa una prueba de laboratorio realizada a $40{ }^{\circ} \mathrm{F}$ y donde se alcanza una resistencia compresiva mayor a 1000 psi después de 24 horas. En la cabeza de pozo luego del flujo de gas superficial se observó cráteres formados en los alrededores de la cabeza de pozo, colocando en entredicho su función estructural.

\subsection{Caso 2. Abandono de pozo}

Pozo con la siguiente configuración 30" a 188 m, 20" @ $428 \mathrm{~m}, 13-3 / 8$ " a 1647,5 m. revestidor de 9-5/8" con desconexión a $972 \mathrm{~m}$. cañoneado cuerpo de arenas en el intervalo 1252-1258 m, se colocó un sistema cementante desde $1647,5 \mathrm{~m}$ hasta $1000 \mathrm{~m}$ y de $900 \mathrm{~m}$ a $650 \mathrm{~m}$, el pozo estaba con flujo de gas leve en la cabeza de pozo, posiblemente en el empaquetador ("pack off") de 13-3/8" x 9-5/8". Se planificó el abandono con retenedor de cemento a $884 \mathrm{~m}$, realizada cementación circulante en el anular de 13-3/8" x 9-5/8" con tope de cemento a $710 \mathrm{~m}$. Cañoneado el revestidor de $9-5 / 8$ " a $327 \mathrm{~m}$. Confirmado tope de cemento a $859 \mathrm{~m}$ dentro del revestidor de $9-5 / 8$ ". Corrido registro de cemento ultra sónico y confirmada buena cementación. Pozo continuando con flujo de gas leve, realizado tapón de cemento desde 360 a 290 m, se inyectó 16 bbls a formación, aguardado 20 horas por fragüe de cemento, verificado tope de cemento a $328 \mathrm{~m}$. Realizado nuevo tapón de cemento desde 328 hasta 228 $\mathrm{m}$, inyectados 6 bbls a formación, verificado tope de cemento a $266 \mathrm{~m}$.

\subsection{Caso 3: Hoyos pilotos}

Para confirmar zonas de probable flujo de gas o agua presurizada superficial son perforados hoyos pilotos de 12-1/4" a una profundidad entre 800 y $1000 \mathrm{~m}$ o a la profundidad de asentamiento de la zapata del revestidor de superficie, que luego son abandonados con tapones de cemento de 200 a $250 \mathrm{~m}$ de longitud cada uno, con un con sistema cementante de peso $14,5 \mathrm{lpg}$, considerando un exceso de $50 \%$, con control de filtrado y es diseñado para ser bombeado por la válvula colocada en el ensamblaje de fondo con un tiempo de fragüe de 6 a 8 horas, otra alternativa es bombearlo vía mecha tricónica sin los jatos, con un tiempo de fragüe de alrededor 12 horas, caso la válvula no abra efectivamente. Si en el pozo piloto se confirma la existencia de una zona presurizada superficial, el diseño del sistema cementante se enfoca principalmente en el desarrollo de fuerza gel en el intervalo de 100 y $500 \mathrm{lbs} / 100$ pies 2 en un intervalo de tiempo menor a 30 minutos 


\section{METODOLOGÍA}

En revestidores donde hay probabilidad de tener zonas presurizadas superficiales bien sea de gas o agua, se recomienda la siguiente metodología:

Si la interpretación sísmica da una probabilidad mediana a alta de tener zonas presurizadas superficiales, tener una política de no perforar pozos en estas zonas, en aquellas zonas donde la probabilidad sea baja, realizar pozos pilotos de diámetros de 12-1/4" para verificar la existencia de estas zonas. Una vez perforada la posible zona de presión anormal con agua de mar, bombear a cada sección colchón viscoso para limpieza del pozo. una vez llegada a la profundidad de estudio, parar circulación por 30 minutos y verificar flujo con ayuda de vehículo operado remotamente, de esta manera se elimina el efecto de densidad equivalente de circulación. Reubicar la locación en una zona que no esté afectada por el registro sísmico por zonas de presión anormal. Realizar un proyecto de pozo con revestidor conductor cementado, caso se confirme la existencia de zonas presurizadas superficiales.

Si el flujo es de agua, realizar un diseño de un sistema cementante cuya diferencia de tiempo entre 100 y 500 lbs $/ 100$ pies $^{2}$ sea menor que 45 minutos. Si el flujo es de gas, realizar un diseño de un sistema cementante cuya diferencia de tiempo entre 100 y $500 \mathrm{lbs} / 100$ pies $^{2}$ sea menor que 30 minutos. En ambos casos utilizar un sistema cementante a base de micro esferas, a fin de obtener mejores propiedades mecánicas con un peso menor.

\subsection{Densidad del sistema cementante}

La densidad del sistema cementante tiene que estar entre los rangos de presión de poro y fractura de las formaciones superficiales que serán perforadas, para zonas de flujo presurizadas superficiales se recomienda la utilización de sistemas cementante a base de micro esferas para utilizar una densidad 12 y $12,5 \mathrm{lpg}$, que sería una solución para la mayoría de los casos. Caso no se utilice un sistema cementante a base de micro esferas se recomienda uno con control de filtrado riguroso con un peso hasta 14,5 lpg, simulaciones son imperativas ejecutarlas, ya que a mayor profundidad de agua en pozos costa afuera, se ejerce mayor presión hidrostática sobre las camadas debajo del lecho marino, teniendo que verificar el peso del sistema cementante para evitar que se alcance el gradiente de fractura.

\subsection{Diseño del sistema cementante}

En general un diseño de un sistema cementante que se utilice en una formación presurizada superficial debe tener:

Antiespumante: su objetivo principal es evitar la generación de espuma durante la mezcla del sistema cementante.

Controlador de filtrado: previenen la deshidratación del sistema cementante y evitan la disminución de la columna hidrostática del mismo debido a la pérdida de agua. Se debe evitar daño a las formaciones productoras, caso existan, entre estos se tienen polímeros orgánicos, látex, entre otros.

Dispersante: se agregan al sistema cementante para proveer propiedades de flujo y permite un bombeo del mismo en flujo turbulento a menor caudal, minimizando los requerimientos de potencia hidráulica.

Retardador: material orgánico, prolongan el tiempo de bombeo y retardan el fraguado del sistema cementante.

Extendedor: se utilizan para reducir la densidad de los sistemas cementantes o para aumentar el rendimiento de estas. La reducción de la densidad persigue disminuir la presión hidrostática de la columna de cemento y prevenir perdidas de circulación debido a rupturas de formaciones débiles.

Bloqueador de gas: aditivos formulados para evitar que el gas atraviese el sistema cementante durante sus etapas de gelificación e hidratación.

La concentración de cada uno de ellos va a depender del aditivo utilizado por cada compañía para alcanzar los objetivos, así como el tiempo de bombeabilidad requerido y la rampa de temperatura y presión simuladas. Siempre de ser posible utilizar la tecnología a base de micro esferas inertes para obtener una baja densidad y buenas propiedades mecánicas del sistema cementante.

Propiedades a verificar en el sistema cementante:

Rápido desarrollo de la resistencia a la compresión, mínimo de 1000 lbs en 24 horas.

$\checkmark$ Control de filtrado riguroso: se utiliza el empleado en gas, entre 30 y $50 \mathrm{cc} / 30$ minutos valores de control de filtrado cercanos a 30 $\mathrm{cc} / 30$ minutos pueden producir un aumento considerable de la viscosidad del sistema cementante. 


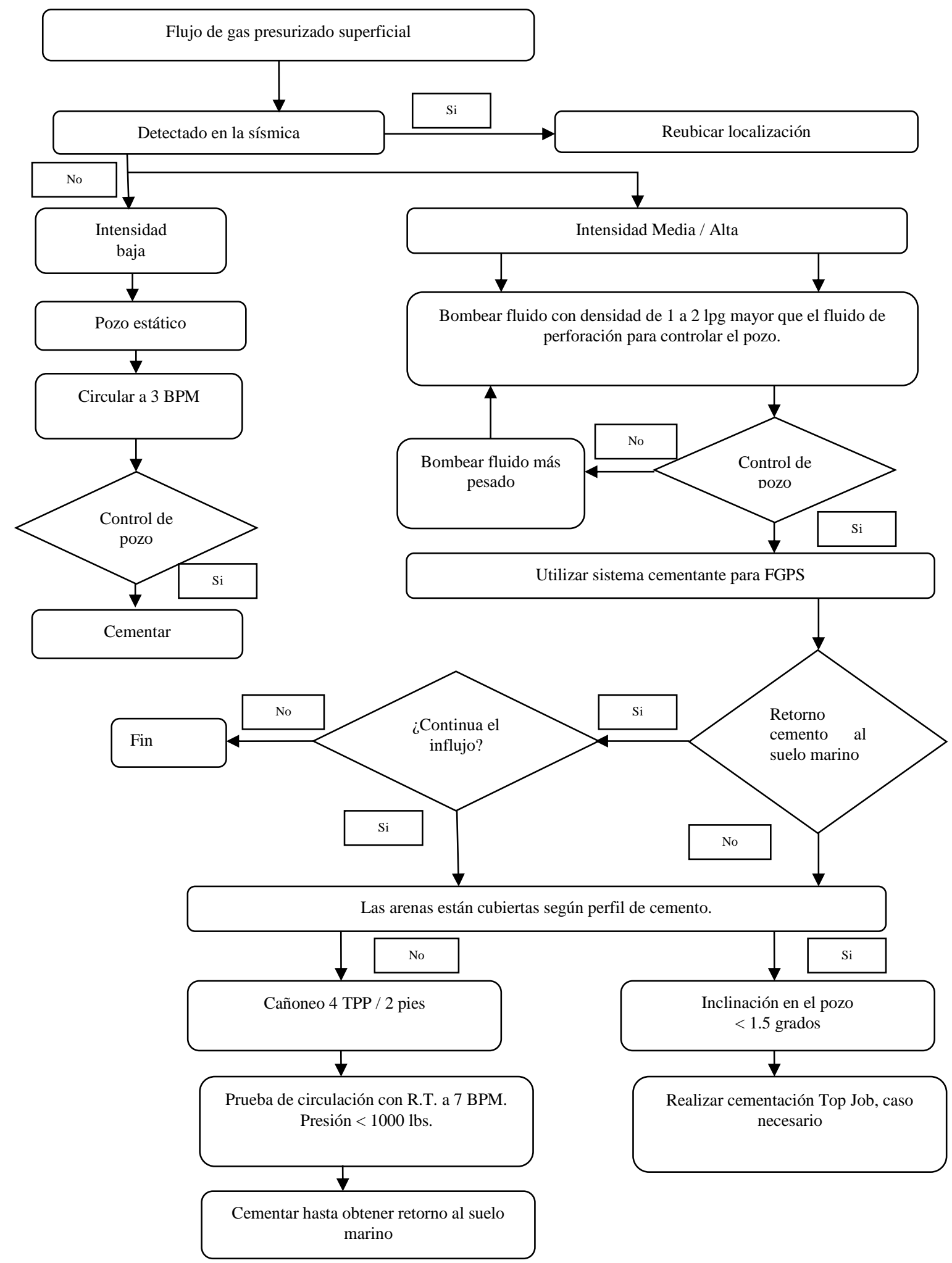

Figura 7: Árbol de decisión: Caso flujo presurizado de gas/agua inesperada. 


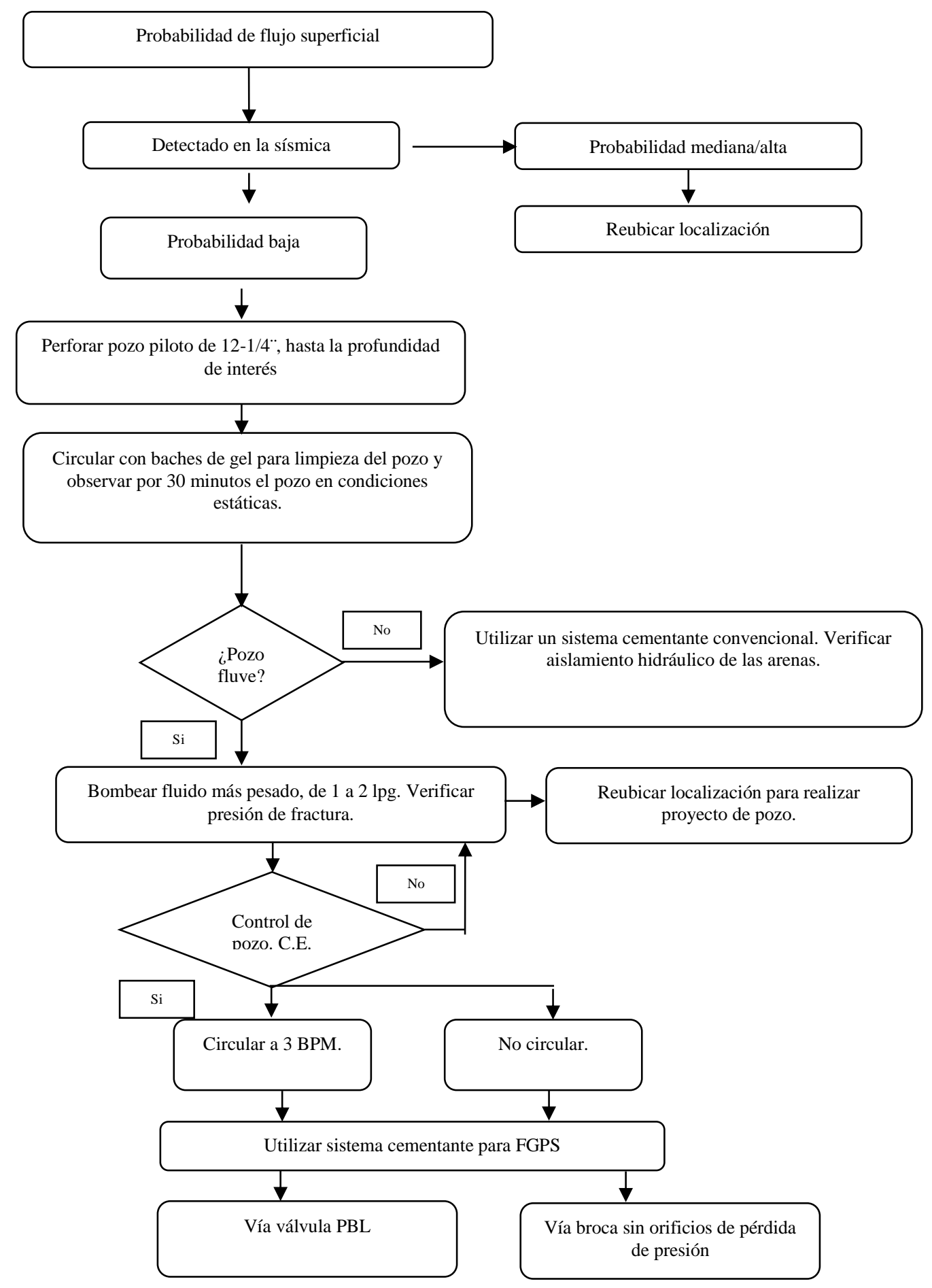

Figura 8. Árbol de decisión: Caso flujo presurizado de gas/agua esperado. Hoyos pilotos. 
Intervalo de tiempo: entre 30 y 45 minutos. la diferencia en tiempo para alcanzar desde 100 a $500 \mathrm{lbs} / 100$ pies 2 de esfuerzo de gel debe estar en el rango de 30 minutos para un flujo severo y 45 minutos para uno moderado o bajo.

$\checkmark \quad$ Agua libre cero.

$\checkmark$ Tiempo de fraguado: entre 4:30 a 6 horas, verificar condiciones de transferencia de cemento, confiabilidad de la bomba y profundidad a realizar la operación. En pozos pilotos el tiempo de bombeabilidad va a depender si la operación va a ser realizada a través de la válvula del ensamblaje de fondo o de la mecha tricónica, normalmente sin jatos, se debe tener disponibilidad de los dos, caso la válvula del ensamblaje de fondo no abra.

$\checkmark \quad$ Simulación hidráulica de los fluidos bombeados como colchones lavadores, espaciadores y sistemas cementantes con volúmenes específicos y caudales para cada uno de ellos para asegurar que en condiciones dinámicas la densidad equivalente de circulación este entre lo gradientes de poro y fractura.

$\checkmark$ Jerarquía reológica entre fluido, colchones y sistema cementante.

\section{RESULTADOS Y ANÁLISIS}

En la tabla 1, se observa la repetibilidad de un diseño de sistema cementante con: antiespumante, reductor $\mathrm{y}$ controlador de filtrado, dispersante, retardador y extendedor, la diferencia de 2 horas y 10 minutos en 50 uc se debe a la muestra de cemento, ya que esta cementación de un revestidor de 13-3/8" slender se ejecuta con cemento de diferentes bateladas.

Debido al que flujo de gas superficial fue inesperado, las pruebas de laboratorio se realizaron de manera rigurosa, por eso observamos en la figura nro. 9 que el desarrollo de 100 a $500 \mathrm{lbs} / 100$ pies $^{2}$ fue en un intervalo de tiempo de 16 minutos y 30 segundos. En un escenario planificado se pudieron ajustar parámetros para que el tiempo de transición estuviera cercano a 30 minutos.

Tabla 1. Repetitividad de un sistema cementante con diferentes bateladas.

\begin{tabular}{|c|c|c|c|c|c|c|c|c|}
\hline $\begin{array}{l}0 \\
\mathbb{D} \\
0\end{array}$ & 总导 导 & 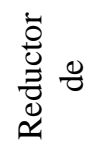 & 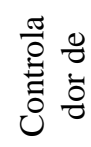 & 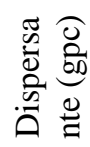 & 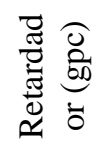 & 总 & 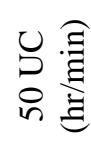 & 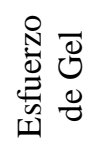 \\
\hline 14 & 0,012 & 0,7 & 0,4 & 0,35 & 0,015 & 2 & 4:00 & 7 \\
\hline 14 & 0,012 & 0,7 & 0,4 & 0,35 & 0,015 & 2 & $6: 10$ & 15 \\
\hline
\end{tabular}

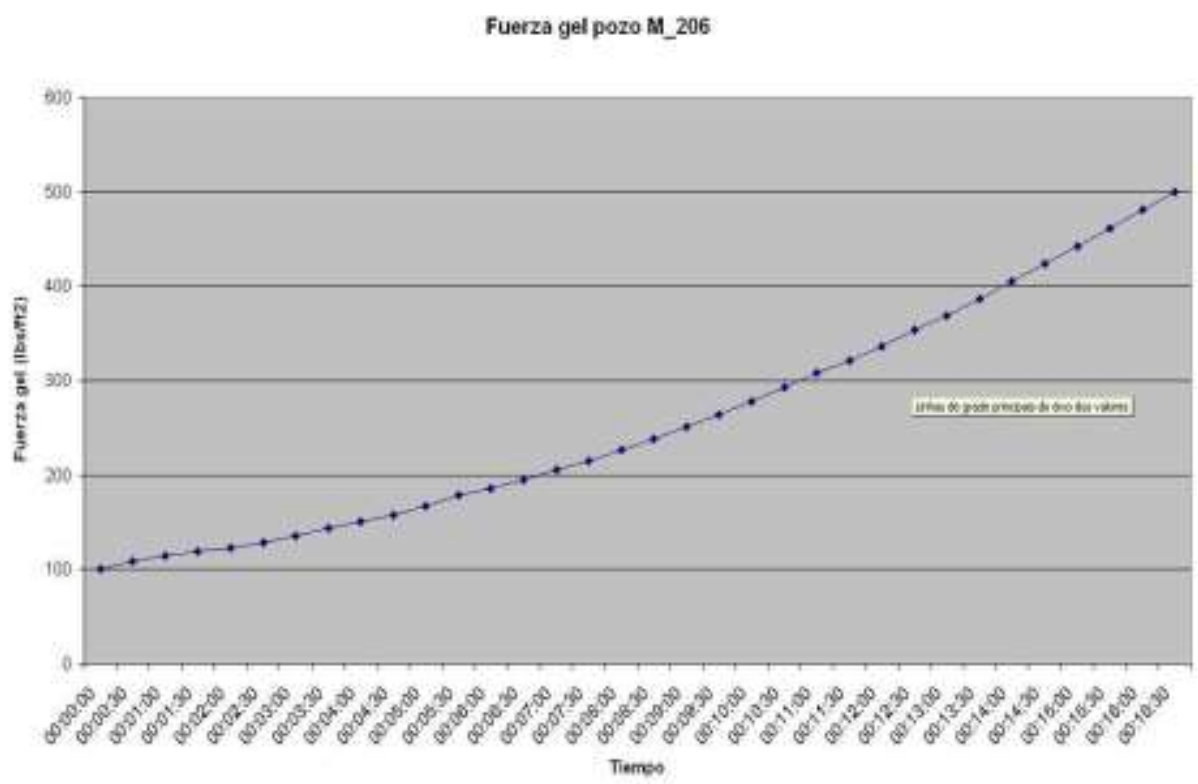

Figura 9. Desarrollo del esfuerzo de gel estatico. Fuente: Prueba de Laboratorio. 
La probabilidad de existencia de zonas presurizadas superficiales es dada a partir de registros sísmicos, que al ser una probabilidad solo se tiene certeza cuando se perfora y se verifica la existencia de estas zonas presurizadas. Debido a esta incertidumbre, se pueden tomar algunas medidas para disminuir el riesgo de perforar en estas zonas con baja probabilidad de ocurrencia.

Como plan a considerar se tiene realizar un diseño de revestidor cuya zapata de superficie quede asentada en una zona arriba de la zona presurizada, de esta manera se perforaría con el equipo de control de pozo y la columna de riser, pudiendo aumentar el peso del lodo, siempre que estemos en una zona competente, la mejor opción para el caso de perforar una zona presurizada es colocar inicialmente en la plataforma un cemento con microesferas y realizar el diseño del sistema cementante con la teoría que explica que se debe alcanzar un esfuerzo de gel en el intervalo del esfuerzo de gel critico a 500 lbs/100 pies $^{2}$ en un tiempo menor a 45 o 30 minutos dependiendo de la severidad del caso.

Antes de ejecutar la cementación:

$\checkmark$ El pozo debe estar bajo control.

$\checkmark \quad$ El fluido debe tener bajo gel.

$\checkmark$ El revoque debe ser fino e impermeable.

$\checkmark$ Remoción de los fluidos de perforación.

$\checkmark$ El revestidor debe tener una buena centralización.

Normalmente el hoyo de superficie se perfora con agua de mar y se bombean píldoras viscosas, para remover los ripios al lecho marino. Las propiedades anteriores son en el caso de que se utilice fluido de perforación, con el equipo de control de pozo y la columna de riser instalados.

\section{CONCLUSIONES}

Flujos de arenas de gas o agua presurizados superficiales no esperados son un riesgo eminente para la seguridad de los trabajadores a bordo, instalaciones y acarrean prejuicios económicos.

La aplicación de este procedimiento y técnica permitió tener un ahorro de 2,3 MM de USD a la compañía, en las fases de los revestidores conductor y de superficie, costos asociados a la etapa de perforación. Los costos asociados debido a la ejecución del sistema cementante fueron de 95 M USD.

La factibilidad de preparación de fluido de mayor peso para controlar el pozo en la etapa de circulación, va a depender de cada unidad marítima de perforación, cuando las condiciones de almacenaje lo permitan. La mayoría de los casos esta opción no pasa el análisis económico, de no tener disponibilidad de realizar fluido de mayor peso para la circulación, evaluar la alternativa de cementar sin circulación previa.

La logística para esperar por los aditivos es complicada y lleva a esperar un tiempo largo para su preparación, en especial en zonas donde no es esperada esta zona de presión anormal.

Para casos identificados de flujo de gas / agua presurizada superficial no esperada de intensidad mediana o alta, caso posible utilizar un sistema cementante a base de micro esferas, que disminuye la densidad del sistema cementante, su permeabilidad, porosidad y posee excelentes propiedades mecánicas.

Tener una política de no perforar en localizaciones donde la interpretación sísmica de una mediana o alta probabilidad de presencia de gas o agua presurizada en profundidades superficiales.

Colocar un sistema cementante a base de micro esferas y agua de mezcla aditivada para alcanzar una resistencia de fuerza gel de $500 \mathrm{lbs} / 100 \mathrm{pies}^{2}$, minimizando la densidad equivalente de circulación (ECD) en el fondo del pozo sin llegar a la presión de fractura. Opción factible si se conoce de antemano la posibilidad de flujo de gas o agua presurizado superficial. Esta opción resulta eficaz a la hora de realizar una cementación en una arena contenedora de agua con presión anormal.

Realizar pozos pilotos de 12-1/4" o 8-1/2" en zonas donde la interpretación sísmica tenga una probabilidad baja de gas presurizado superficial, ya que prevé una logística mejor de control, circulación y cementación de pozo.

Analizar la factibilidad de realizar proyectos de perforación convencional: conductor de 30" cementado, superficial de 20", perforar la zona de presión anormal con equipamiento de control de pozo y columna de riser, aunque esta opción requiera de una fase adicional.

Analizar la posibilidad de utilización de la tecnología de un sistema cementante espumado, a base de nitrógeno, muy utilizado en el golfo de México, ya que esta tecnología permite el diseño de pesos de sistemas cementantes inferiores a 11,5 lpg, caso no se observen zonas productoras críticas. 


\section{AGRADECIMIENTOS}

Al departamento de cementación y revestimiento. Sercim, Macae, Rio de Janeiro. Petrobras por permitir el estudio de este proyecto.

A la Universidad Central de Venezuela.

A la Escuela de Ingeniería Mecánica de la Universidad Central de Venezuela.

Al departamento de Post Grado de la Facultad de Ingeniería de la Universidad Central de Venezuela.

\section{NOMENCLATURA}

Bbl: Barriles.

BPM: Barriles por minutos.

cc: $\quad$ Centímetros cúbicos.

${ }^{\circ} \mathrm{F}$ : $\quad$ Grados Fahrenheit.

FGPS: Flujo de Gas Presurizado Superficial.

gpc: Galones por pie cúbico.

lpg: Libras por galón.

lb: Libras.

m: Metros.

mD: Mili Darcys.

psi: Libras por pulgadas cuadradas.

pies $^{2}$ : Pies al cuadrado.

sc: $\quad$ Sacos

TPP: Tiros Por Pie.

\section{REFERENCIAS}

[1] Arshad W., M. Cockram, Rashpetco and M. Bahr, "Foam Cementing Controls Deepwater Shallow Flow in the East Mediterranean". SPE Nro 74402. 2002.

[2] Dan T. Mueller. "Redefining the Static Gel Strength Requirements for Cements Employed in SWF Mitigation ". 14282-MS. 2002

[3] Guevara I., L. Marcano, F. Paz. "Cementaciones Superficiales con Gas en Pozos Costa Afuera. Proyecto Plataforma Detana". SEFLU CEMPO. 2006.

[4] J. León, O. Ortiz, F. Guarin, C. Díaz. "Variables que influyen en un proceso de inyección cíclica de diluyente en formación para el incremento de la productividad de pozos de crudo extrapesado." Revista UIS Ingenierías12.2 Jul - Dic. 2013.

[5] Lopez J., D. Garrett, A. Zanaty, and A. Waheed. "Successful Mitigation of Deepwater Shallow Flows in the East Mediterranean Region - Case Histories". SPE nro. 120399. 2009.
[6] Luiz Alberto Santos Rocha. Cecilia Toledo de Azevedo. Projetos de poços de petróleo. Editora interciencia. Petrobras. Rio de Janeiro. Brasil. 2007.

[7] Mammadbayli R, J. Greener, D. Doherty, J. Reyes, P. Torres "Foamed Cement successfully applied in shallow water environment in Caspian Sea. 2006.

[8] Mavares, Francisco J y Pertuz, Alberto D. Diseño de un sistema cementante para pozos utilizados en anclaje de plataformas de producción en aguas profundas: Caso: Río de Janeiro, Brasil. Rev. Fac. Ing. UCV. 2013, vol.28, n.1, pp. 73-81

[9] Mueller, Dan T, "Redefining the Static Gel Strength Requirements for Cements Employeed in SWF mitigation" Offshore Technology Conference nro. 14282. 2002.

[10] Quiroga-Jordan Elizabeth, Calvete-Gozález, Fernando and Ortiz-Cancini Olga P., "Influencia de la extracción de fluidos de control antes de realizar una prueba de ascenso de presión en un pozo." Revista UIS Ingenierías 12.2. 2014

[11] Pederson R., T. Oian, T. Haugland, B. Watts, S. Taoutaou, A. Stakkestad. () "A Successful Field Experience: Shallow Water Flow - How to Avoid the Need for an Additional Casing String. IADC/SPE 112716.

[12] Sabins, FL., et al., "Transition Time of Cement Slurries Between the Fluid and Set State". SPE 9285. 1980.

[13] Schuberth P. and M. Walker. "Shallow Water Flow Planning and Operations: Titan No. 1 Exploration Well, Deepwater Gulf of Mexico". SPE/IADC Nro 65751. 2000. 